\title{
The PINGU detector
}

\author{
Thomas Ehrhardt* for the IceCube-Gen2 collaboration ${ }^{\dagger}$ \\ Universität Mainz, Germany \\ E-mail: tehrhardtaicecube.wisc.edu
}

\begin{abstract}
The world's largest neutrino telescope, the IceCube Neutrino Observatory, is built in one of the planet's most extreme environments: at South Pole Station Antarctica. Completed in 2010, and instrumenting about a cubic-kilometre of ice, IceCube has been designed to measure the flux of astrophysical neutrinos it recently discovered. It also comprises a low-energy detector array, called DeepCore, that has performed world-leading indirect dark matter searches and very high statistic studies of atmospheric neutrinos down to approximately $10 \mathrm{GeV}$. Building on the success of DeepCore, a new infill array called PINGU (Precision IceCube Next Generation Upgrade) is now being proposed that will further reduce the in-ice energy threshold to a few GeV. Such a detector will be capable of significantly expanding the current low-energy program, including the potential to make a first determination of the neutrino mass ordering. Here we discuss the design and sensitivity of the PINGU detector.
\end{abstract}

The European Physical Society Conference on High Energy Physics

22-29 July 2015

Vienna, Austria

\footnotetext{
${ }^{*}$ Speaker.

†http://icecube.wisc.edu/collaboration/authors/icecubegen2_2015
} 


\section{Introduction}

Three-flavour neutrino oscillations have become a widely accepted effect by now. Here, massive neutrinos come with both mass and flavour eigenstates, which are rotated versions of each other, connected by the $3 \times 3$ neutrino mixing matrix [1]. When a neutrino is produced in a certain flavour eigenstate, $\alpha$, there is a non-vanishing probability $P_{\alpha \rightarrow \beta}$ that it will be detected as a different flavour eigenstate, $\beta$, after having travelled a distance $L$.

First clear evidence for this quantum mechanics phenomenon, which successfully explains both the so-called "solar neutrino problem" as well as the atmospheric neutrino anomaly, was found by Super-Kamiokande in 1998 [2], through the measurement of the zenith angle distribution of atmospheric neutrinos. Since then, experiments detecting neutrinos from other sources, such as particle accelerators, nuclear reactors, and the Sun, have contributed to solidifying the 3-flavour oscillation scenario and constraining the free parameters of the mixing matrix. Still largely unknown as of today is the value of the CP-violating phase $\delta_{\mathrm{CP}}$.

Also still to be resolved is the question of the neutrino mass hierarchy, i.e. the sign of the squared mass difference $\Delta m_{32}^{2}$ between the second and third mass eigenstate. The hierarchy is referred to as "normal" if $\Delta m_{32}^{2}>0$, i.e. if the third mass eigenstate is the heaviest, and as "inverted" otherwise. The fact that the 1-3 mixing angle $\theta_{13}$ has been found to be rather large [3] makes it conceivable that huge megaton-scale atmospheric neutrino detectors such as the planned Precision IceCube Next Generation Upgrade (PINGU) [4] will allow for an unambiguous determination of the mass hierarchy on relatively short time scales, by exploiting matter effects in the Earth that characteristically alter the oscillation probabilities. Apart from neutrino oscillation measurements, the physics reach of PINGU includes the indirect search for dark matter as well as the detection of supernova neutrinos. In addition, PINGU will explore the possibility of using neutrinos for the first time in order to conduct Earth tomography studies.

This document first introduces the existing IceCube detector and the layout and design of PINGU. We then focus on the measurement of the mass hierarchy and discuss the predicted sensitivity, before presenting the ability of PINGU to precisely determine the "atmospheric" oscillation parameters $\theta_{23}$ and $\Delta m_{31}^{2}$, as well as its performance at conducting precision tests of the standard 3-flavour $v_{\tau}$ appearance prediction.

\section{From IceCube to PINGU}

The IceCube Neutrino Observatory [5], constructed at the location of the Amundsen-Scott South Pole Station in Antarctica, is a cubic-kilometre Cherenkov neutrino detector, instrumenting the glacial ice at depths between $1450 \mathrm{~m}$ and $2450 \mathrm{~m}$. 5160 digital optical modules (DOMs) - glass pressure spheres enclosing one downward-facing $10^{\prime \prime}$ PMT each - are distributed on 86 strings, eight of which are part of the more densely instrumented DeepCore array, with inter-string spacings ranging from around $40 \mathrm{~m}$ up to $70 \mathrm{~m}$, compared to a typical spacing of $125 \mathrm{~m}$ in IceCube (cf. Fig. 1).

IceCube is designed to detect the interactions of high-energy neutrinos, and has already discovered a neutrino flux that is of astrophysical origin [6], whereas DeepCore is sensitive to maximum 
disappearance of muon neutrinos at an energy of around $E_{v}=25 \mathrm{GeV}$, and has thereby proven to be able to provide competitive measurements of the atmospheric oscillation parameters [7].

Making use of an increased PMT density, and closely following the design of DeepCore, PINGU will lower the neutrino detection threshold of the whole array even further, down to a few GeV. Extensive geometry optimisation studies have resulted in a modified detector layout compared to the one presented in [4]: 40 additional strings will be deployed within the lower part of DeepCore, where the ice properties are most beneficial to the detection of the Cherenkov light. The strings will be spaced apart $22 \mathrm{~m}$ horizontally on average, and they will be equipped with 96 optical modules each, with a vertical spacing of only $3 \mathrm{~m}$. The predicted fractional neutrino energy resolution with this detector configuration amounts to around $20 \%$ for energies exceeding $10 \mathrm{GeV}$, and the uncertainty on the cosine of

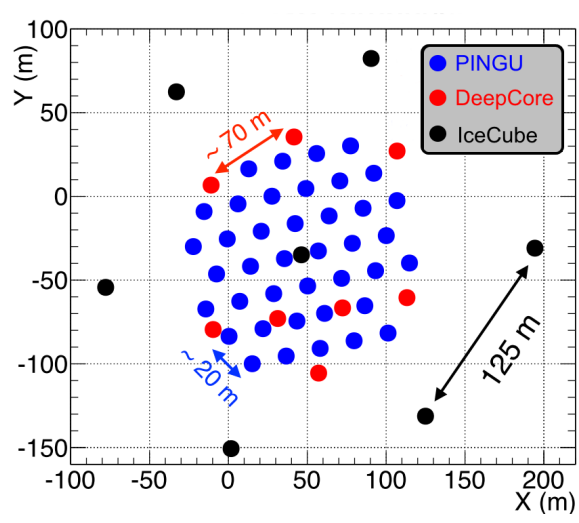

Figure 1: Top-down view of the proposed PINGU detector. The coordinates of the closest IceCube and the DeepCore strings are depicted in black and red, respectively. the reconstructed zenith angle is about 0.15 at $10 \mathrm{GeV}$, improving with increasing energy.

While dedicated calibration devices will be making in situ measurements of detector related systematics, due to its location, the PINGU sub-array will allow extending the active veto capabilities demonstrated by DeepCore [7], and thus further enhance the atmospheric muon background rejection.

\section{Neutrino Oscillation Physics with PINGU}

\subsection{Neutrino mass hierarchy}

The design of the PINGU detector is adapted to one of its main physics goals: the decisive determination of the neutrino mass hierarchy. This can be revealed via the measurement of matter effects altering the oscillation probabilities of the vacuum regime as atmospheric neutrinos traverse the Earth. Through the MSW effect [8,9], resonant enhancement of the $v_{\mu}$ survival and $v_{e} \leftrightarrow v_{\mu}$ transition probabilities will occur at energies below $10 \mathrm{GeV}$ if the hierarchy is normal, while the corresponding anti-neutrino channels are affected if the inverted hierarchy is realised in nature. In addition, "parametric enhancement" [10] occurs due to a jump in the density profile at the boundary between the Earth's mantle and core [11]. Which mechanism dominates depends on the neutrino's zenith angle $\theta_{v}$ as well as on its energy $E_{v}$, with resonant enhancement playing a role for neutrinos that only cross the Earth's mantle, i.e. when $\cos \theta_{v} \gtrsim-0.84$. For energies exceeding $15 \mathrm{GeV}$, the probability for both electron neutrinos and anti-neutrinos to oscillate into their muon flavour counterparts becomes increasingly small, and the different mass hierarchies become less and less distinguishable [10].

Even though, to first order, the matter effect is symmetric under the exchange of both hierarchy and charge $(v \leftrightarrow \bar{v})$, charge discrimination is not strictly necessary in order to determine the hierarchy. The atmospheric flux of neutrinos exceeds that of anti-neutrinos by some $10 \%$, and the 

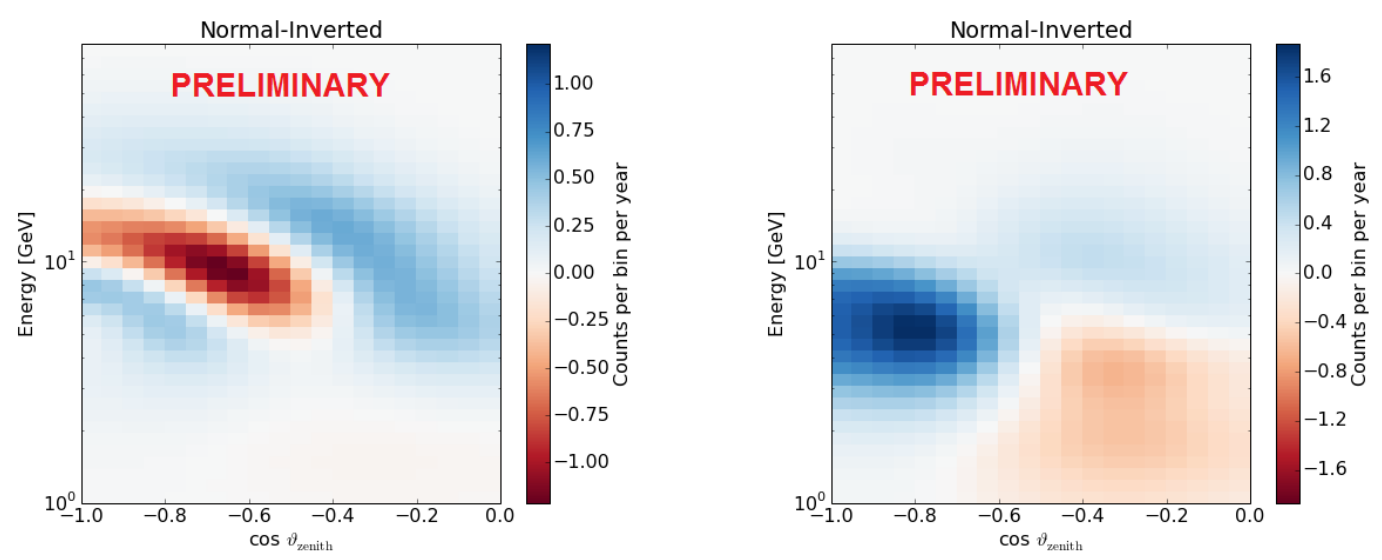

Figure 2: Difference in annual event rates between the normal and inverted hierarchy after background rejection cuts, for events classified as track-like (left) and cascade-like (right).

neutrino cross-sections are approximately a factor two larger than those of anti-neutrinos, resulting in a net asymmetry of count rates at the detector. It does however explain the need for a megatonscale detector to reach sufficient event statistics, so that the few percent residuals in the sensitive region can be resolved.

Fig. 2 shows the difference in annual event rate between the normal and inverted hierarchy for track- and cascade-like events after rejection cuts in PINGU, binned in cosine of the reconstructed neutrino zenith angle and reconstructed neutrino energy. Clearly, despite the dilution brought about by limited angular and energy resolution, there are disjunct regions that exhibit either higher or lower rates under each of the hierarchies, and which permit determination of the hierarchy - provided good control of systematic effects.

\subsubsection{Sensitivity}

We have developed two different methods to evaluate the sensitivity of PINGU to the neutrino mass hierarchy. The first one makes use of a large ensemble of Poisson-fluctuated pseudoexperiments drawn from a set of fiducial parameters within each hierarchy, in order to determine the median sensitivity $\alpha$, with second kind error probability $\beta=0.5$. Here, for each pseudoexperiment we determine the best fitting parameter values for both hierarchies in order to find the median log-likelihood ratio (LLR) under the assumed true hierarchy. The parameters of the alternative hierarchy that best fit the assumed true hierarchy hypothesis are used to obtain the second LLR distribution. This distribution then allows to estimate the probability of misidentifying the true hierarchy, which is converted into the significance of rejecting the other hierarchy.

The second approach employs the " $\overline{\Delta \chi^{2}}$ " method often encountered in the literature, which corresponds to the sum of the weighted squared differences between the expected experimental outcomes under the two hierarchies, minimised over the parameters within the opposite hierarchy. Typically, it is evaluated as a $\chi^{2}$ with 1 degree of freedom, a valid approach if the relevant distribution is normal [12].

The median sensitivity obtained in the LLR approach, transformed into a corresponding number of Gaussian standard deviations, is shown as a function of exposure of the fully completed detector in the left panel of Fig. 3 for the current global best fit values of $\theta_{23}$ and $\Delta m_{31}^{2}$ [13]; it 

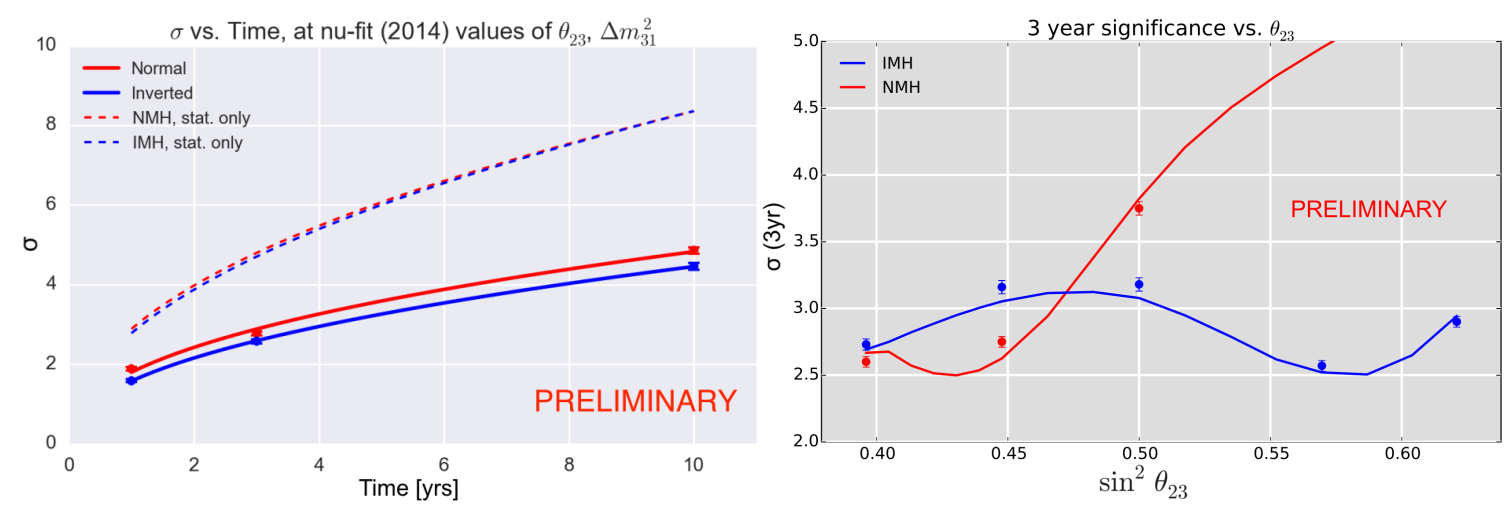

Figure 3: PINGU neutrino mass hierarchy sensitivity, with individual points obtained with the LLR method, vs. years of data taking with the full detector (left), and $\sin ^{2} \theta_{23}$ (right). In the latter case, the lines stem from the "$\overline{\Delta \chi^{2}} "$ approach. See text for details.

reaches $3 \sigma$ after slightly more than three years of data, regardless of the hierarchy. The right panel, on the other hand, shows the dependence of the sensitivity on $\sin ^{2} \theta_{23}$, after three years of data taking. The normal hierarchy together with $\sin ^{2} \theta_{23}>0.5$ would be most beneficial to the hierarchy determination because the matter induced asymmetry is strongest and the rates are highest in this configuration. For smaller values of $\theta_{23}$ in the normal hierarchy, second octant solutions in the inverted hierarchy result in more compatible fits. Note that while the points were obtained with the LLR method, the remainder of the parameter space was probed using the faster $\overline{\Delta \chi^{2}}$ approach.

Apart from the atmospheric oscillation parameters $\theta_{23}, \theta_{13}$ and $\Delta m_{31}^{2}$, the results presented here include the following systematic effects: parameters describing errors in the energy scale and the overall flux/effective area, the ratio of $v_{e}$ to $v_{\mu}$ present in the atmospheric flux, the flux spectral index, and one systematic covering uncertainty of both the $v$ to $\bar{v}$ flux ratio and their relative crosssections. For $\theta_{23}$ and $\Delta m_{31}^{2}$, no prior knowledge is assumed, while e.g. the energy scale has a $10 \%$ Gaussian prior on it, which is based on recent detector calibration data. In addition, studies involving detailed neutrino-nucleon interaction parameters [14] and a large number of atmospheric flux systematics have been and are being carried out, finding only small impact on the sensitivity.

\subsection{Atmospheric oscillation parameters}

With its low energy threshold, the PINGU detector also has good sensitivity to the neutrino oscillation parameters, most notably the atmospheric parameters $\theta_{23}$ and $\Delta m_{31}^{2}$, currently known with $3 \sigma$ relative precision of $32 \%$ and $11 \%$, respectively [13]. For a given set of true values under each neutrino mass hierarchy, the likelihood-based analysis presented in the previous section, taking into account both the cascade- and track-like channel and assuming that the hierarchy is correctly identified, yields the expected constraints on the atmospheric parameters. These are shown for 3 years of exposure and the normal hierarchy in the left panel of Fig. 4.

\section{$3.3 v_{\tau}$ appearance}

PINGU's ability to perform a measurement of $v_{\tau}$ appearance will complement the neutrino mass hierarchy and atmospheric oscillation parameter measurements described above. While the OPERA experiment has discovered $v_{\tau}$ appearance at larger than $5 \sigma$ significance with the recent 

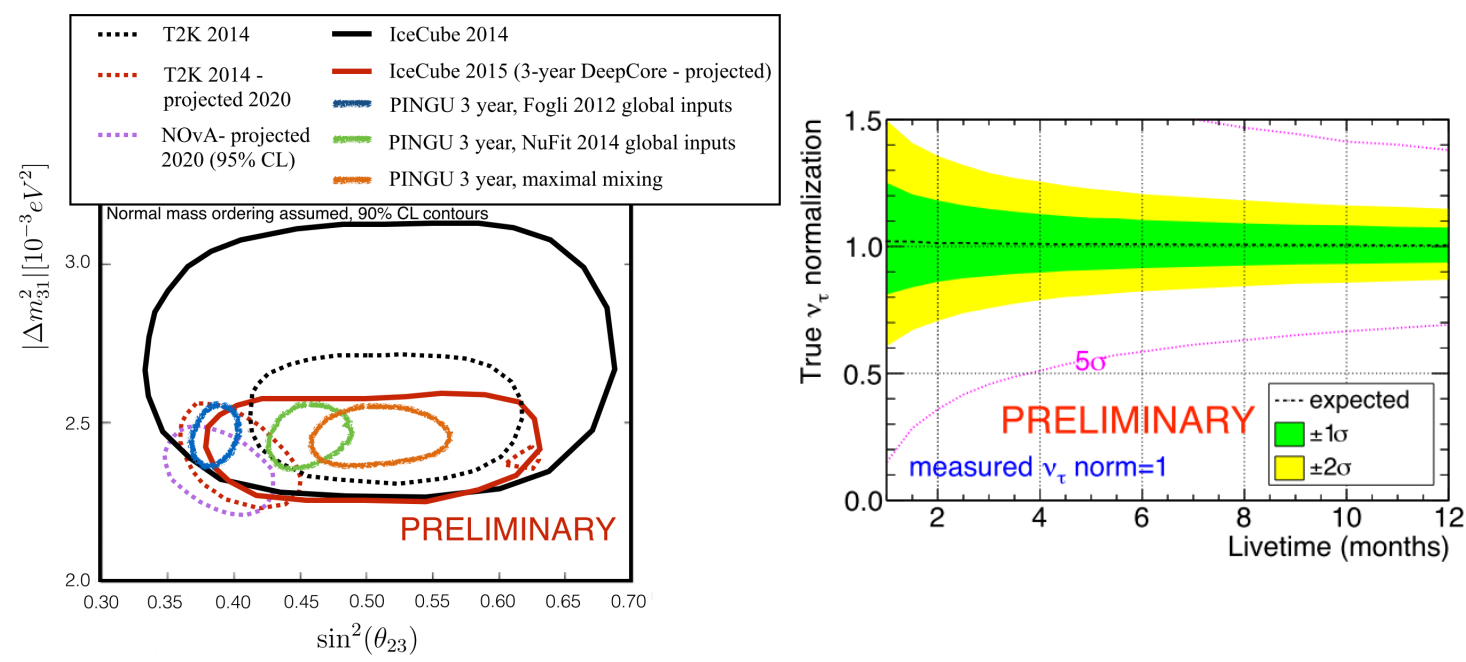

Figure 4: Left: Projected PINGU constraints (90\% C.L.) on atmospheric oscillation parameters after 3 years of exposure, with existing results from IceCube/DeepCore and (projected) sensitivities from T2K and NOvA. Right: PINGU $v_{\tau}$ appearance normalisation precision versus exposure time. See text for details.

detection of a fifth $v_{\tau}$ candidate event [15], PINGU can yield further knowledge about the validity of the current standard 3-flavour oscillation model, by a comparison between the rate of $v_{\tau}$ appearance to that of disappearance of $v_{\mu}$ in the atmospheric neutrino flux. Non-compatibility between these would be a clear indication of new physics, brought about e.g. by non-unitarity of the leptonic mixing matrix due to the existence of sterile neutrinos or non-standard interactions.

Using the same atmospheric muon rejection criteria as the mass hierarchy analysis, but selecting a purer sample of events classified as cascade-like, a preliminary study has been performed that searches for $v_{\tau}$ appearance in PINGU. In this sample, $1.7 \mathrm{k} v_{\tau}$-induced events are expected per year, with a background of $11.2 \mathrm{k}$ events. Since no $v_{\tau}$ are present in the unoscillated flux, the signal is concentrated in the upgoing region, while the background is concentrated closer to the horizon. A statistically significant measurement of $v_{\tau}$ appearance is possible even though the background dominates in the upgoing region, and a $10 \%$ precision on the $v_{\tau}$ normalisation is achievable after six months of exposure, as depicted in the right panel of Fig. 4.

\section{Conclusion}

PINGU's low neutrino detection energy threshold will make accessible the signature of the still unknown hierarchy of neutrino masses, enable a precision measurement of neutrino oscillation parameters, and will allow searching for dark matter particles of low mass. As an integral part of the planned upgrades to the IceCube neutrino observatory, the PINGU detector will thus greatly enhance the reach of the firmly established IceCube/DeepCore particle physics program.

\section{References}

[1] Z. Maki, M. Nakagawa and S. Sakata, Prog. Theor. Phys. 28, 870 (1962)

[2] Y. Fukuda et al. (Super-Kamiokande Collaboration), Phys. Rev. Lett. 81, 1562 (1998) 
[3] F. P. An et al. (DayaBay Collaboration), Phys. Rev. Lett. 112, 061801 (2014)

[4] M. G. Aartsen et al. (IceCube-PINGU Collaboration), [arXiv: 1401 . 20 46v1]

[5] IceCube Collaboration homepage, http://icecube.wisc.edu/

[6] M. G. Aartsen et al. (IceCube Collaboration), Phys. Rev. Lett. 113, 101101 (2014)

[7] M. G. Aartsen et al. (IceCube Collaboration), Phys. Rev. D 91, 072004 (2015)

[8] L. Wolfenstein, Phys. Rev. D 17, 2369 (1978)

[9] S. P. Mikheyev, A. Y. Smirnov, Il Nuovo Cimento C 9, 17 (1986)

[10] E. K. Akhmedov, S. Razzaque and A. Y. Smirnov, JHEP 1302, 082 (2013)

[11] A. M. Dziewonski and D. L. Anderson, Physics of the Earth and Planetary Interiors 25, 297 (1981)

[12] M. Blennow, P. Coloma, P. Huber and T. Schwetz, JHEP 03, 028 (2014)

[13] M. C. Gonzalez-Garcia, M. Maltoni and T. Schwetz, JHEP 11, 052 (2014)

[14] S. Böser (for the IceCube-PINGU Collaboration), to appear in the Proceedings of NEUTEL 2015, XVI International Workshop on Neutrino Telescopes (Venice, Italy, 2015)

[15] N. Agafonova et al. (OPERA Collaboration), Phys. Rev. Lett. 115, 121802 (2015) 